\title{
Contributor Index
}

\section{Volume 47, 2003}

Subject index on page 1164 .

\section{A}

Aaberg TM Jr (see Grossniklaus et al). 2003;47:239-246

Abascal-Agorreta M (see Vera-Alvarez et al). 2003;47: $1107-1110$

Abdul-Karim FW (see Abramovich et al). 2003;47:405409

Abdul-Karim FW (see Wen et al). 2003;47:135-140

Abe K (see Sakaki et al). 2003;47:321-324 (Letter)

Abramovich CM (see Wen et al). 2003;47:135-140

Abramovich CM, Wasman JK, Siekkinen P, Abdul-

Karim FW. Histopathologic Correlation of Atypical

Parakeratosis Diagnosed on Cervicovaginal Cytology. 2003;47:405-409

Abrari A (see Sherwani et al). 2003;47:506-508

Abu-Jawdeh GM (see Ayata et al). 2003;47:973-978

Afify AM, Al-Khafaji BM, Kim B, Scheiman JM. Endoscopic Ultrasound-Guided Fine Needle Aspiration of the Pancreas: Diagnostic Utility and Accuracy. 2003; 47:341-348

Agarwal A (see Anand et al). 2003;47:467-469

Agarwal PK (see Goel et al). 2003;47:1119-1122

Agarwal S (see Goel et al). 2003;47:1119-1122

Aggarwal N (see Mishra et al). 2003;47:1001-1007

Ahn S-H (see Jun et al). 2003;47:685-687

Aisner S (see Das et al). 2003;47:197-201

Al Reyees FA (see El Hag et al). 2003;47:387-392

AlAnsari AG (see Gupta et al). 2003;47:393-398

Alberti Masgrau N (see Vaillo Vinagre et al). 2003;47: 485-489

Alexis N (see Ghio and Alexis). 2003;47:695-696 (Letter)

Ali SZ (see Allen et al). 2003;47:183-187

Ali SZ (see Parwani et al). 2003;47:325-331

Al-Khafaji BM (see Afify et al). 2003;47:341-348

Allen EA, Parwani AV, Siddiqui MT, Clark DP, Ali SZ. Cytopathologic Findings in Breast Masses in Men with HIV Infection. 2003;47:183-187

Alonso de Ruiz P (see Castro-Gómez et al). 2003;47:590-594

Álvarez C (see Ortiz-Rey et al). 2003;47:490-494

Alvarez T (see Arias et al). 2003;47:1145-1146 (Letter)

Amanjit (see Mohan et al). 2003;47:792-794

Amirtham U (see Madhumathi et al). 2003;47:101-103 (Letter)
Anand M, Kumar R, Jain P, Gupta R, Ghosal N, Sharma A, Agarwal A, Sharma MC. Metastatic Anaplastic Oligodendroglioma Simulating Acute Leukemia: A Case Report. 2003;47:467-469

Anbo J (see Kikuchi et al). 2003;47:495-500

Andorno S (see Omodeo-Zorini et al). 2003;47:985-990

Angeletti C. Application of Proteomic Technologies to Cytologic Specimens: A Review. 2003;47:535-544

Angeli K (see Oregioni et al). 2003;47:98-99 (Letter)

Antón I (see Ortiz-Rey et al). 2003;47:490-494

Arai T (see Jobo et al). 2003;47:611-615

Araki H (see Kobayashi et al). 2003;47:1069-1073

Arguelles R (see Lerma et al). 2003;47:575-580

Arias LF, Alvarez T, Gonzalez L, Mendoza ML, Blanco J, Garcia-Asenjo JL, Prats D. BK Viral Infection in Kidney Transplantation: Importance of "Decoy" Cells. 2003;47:1145-1146 (Letter)

Ariga R (see Lal et al). 2003;47:951-959

Arora VK (see Singh et al). 2003;47:368-372

Arora VK (see Singh et al). 2003;47:960-964

Arora VK, Singh N, Chaturvedi S, Bhatia A. Significance of Cytologic Criteria in Distinguishing Small Cell from Non-Small Cell Carcinoma of the Lung. 2003;47:216-220

Ayata G, Abu-Jawdeh GM, Fraser JL, Garcia LW, Upton MP, Wang HH. Accuracy and Consistency in Application of a Probabilistic Approach to Reporting Breast Fine Needle Aspiration. 2003;47:973-978

B

Baehner F, Sudilovsky D. Fine Needle Aspiration Cytology of Intraoral Epithelioid Hemangioma: A Report of Two Cases. 2003;47:275-280

Bagué S (see Lerma et al). 2003;47:575-580

Bahar K (see Soyuer et al). 2003;47:581-589

Ballestín Carcavilla C (see Vaillo Vinagre et al). 2003;47: 485-489

Baloch Z (see Nassar et al). 2003;47:313-315 (Letter)

Balu S (see Premalata et al). 2003;47:475-479

Bamba M (see Kobayashi et al). 2003;47:103-106 (Letter) 
Baracat EC (see Mattosinho de Castro Ferraz et al). 2003; 47:154-158

Barroca HMMT, Gil-da Costa, Carvalho JL. Cytologic Profile of Rhabdoid Tumor of the Kidney: A Report of 3 Cases. 2003;47:1055-1058

Basir Z, Pello N, Dayer AM, Shidham VB, Komorowski RA. Accuracy of Cytologic Interpretation of Pancreatic Neoplasms by Fine Needle Aspiration and Pancreatic Duct Brushings. 2003;47:733-738

Bastert G (see Melsheimer et al). 2003;47:124-128

Basu D, Nilkund J. Detecting Mycobacteria in Romanowsky-Stained Cytologic Smears: A Case Report. 2003;47:774-776

Bauchman G (see Yarkin et al). 2003;47:450-456

Bédard YC, Pollett AF, Leung SW, O'Malley FP. Assessment of Thin-Layer Breast Aspirates for Immunocytochemical Evaluation of HER2 Status. 2003;47:979-984

Belinson JL (see Pan et al). 2003;47:45-50

Benevenia J (see Das et al). 2003;47:197-201

Benigni E (see Omodeo-Zorini et al). 2003;47:985-990

Bentz JS (see Rowe et al). 2003;47:602-604

Bergeron C, Fagnani F. Performance of a New, LiquidBased Cervical Screening Technique in the Clinical Setting of a Large French Laboratory. 2003;47:753-761

Berlière M (see Weynand et al). 2003;47:149-153

Bernard P (see Weynand et al). 2003;47:149-153

Berry M (see Rowe et al). 2003;47:602-604

Bettini JdSR, Soares EG, Duarte G, Simões RT, Simões AL. PCR Diagnosis of HPV in Cervical Biopsies of CIN and Invasive Neoplasia Formerly Diagnosed as HPV Negative. 2003;47:545-549

Bhat S (see Kini and Bhat). 2003;47:113-114 (Letter)

Bhatia A (see Arora et al). 2003;47:216-220

Bhatia A (see Singh et al). 2003;47:368-372

Bhatia A (see Singh et al). 2003;47:960-964

Bisi H (see Longatto-Filho et al). 2003;47:317-318 (Letter)

Biswas S (see Premalata et al). 2003;47:475-479

Blanco J (see Arias et al). 2003;47:1145-1146 (Letter)

Blázquez S (see Mayayo et al). 2003;47:1116-1118

Boavida J (see Cavaco et al). 2003;47:5-12

Boldorini R (see Omodeo-Zorini et al). 2003;47:985-990

Bonifacio-Gori D (see DellaGiustina et al). 2003;47:10171022

Boob R (see Geramizadeh et al). 2003;47:701-702 (Letter)

Bortolan J (see Longatto-Filho et al). 2003;47:317-318 (Letter)

Brahmi U, Srinivasan R, Komal HS, Joshi K, Gupta SK, Rajwanshi A. Comparative Analysis of Electron Microscopy and Immunocytochemistry in the Cytologic Diagnosis of Malignant Small Round Cell Tumors. 2003;47:443-449

Bunyaratavej K (see Tanboon et al). 2003;47:1143-1144 (Letter)

Bur ME (see Cao et al). 2003;47:1033-1037

Burchett IJ (see Loo and Burchett). 2003;47:83-87

Burger PC (see Parwani et al). 2003;47:325-331
Burns DK (see Raisanen et al). 2003;47:518-520 (Letter)

Burstein D (see Yarkin et al). 2003;47:450-456

\section{C}

Calvin C, Sandweiss L, Wallace WD. Alternative Fixation Techniques for Conventional Pap Smears. 2003; 47:1139-1140 (Letter)

Canda MS (see Topal et al). 2003;47:320-321 (Letter)

Cangiarella J, Waisman J, Simsir A. Cytologic Findings with Histologic Correlation in 43 Cases of Mammary Intraductal Adenocarcinoma Diagnosed by Aspiration Biopsy. 2003;47:965-972

Cao QJ, Panetti C, Marconi S, Krebs P, Lorenzana RR, Goulart RA, Bur ME. Vimentin and Leukocyte Common Antigen-Negative Molding Cells in Pleural Effusions of Patients with Small Cell Lung Carcinoma. 2003;47:1033-1037

Caraway NP, Fanning CV, Stewart JM, Tarrand JJ, Weber KL. Coccidioidomycosis Osteomyelitis Masquerading as a Bone Tumor: A Report of 2 Cases. 2003; 47:777-782

Carreras AM (see Lerma et al). 2003;47:575-580

Carvalho JL (see Barroca et al). 2003;47:1055-1058

Castro WH (see Guimarães et al). 2003;47:1074-1076

Castro-Gómez L, Córdova-Ramírez S, Duarte-Torres R, Alonso de Ruiz P, Hurtado-López LM. Cytologic Criteria of Cystic Papillary Carcinoma of the Thyroid. 2003;47:590-594

Catalina-Fernandez I (see Sáenz-Santamaría et al). 2003; 47:1-4

Catalina-Fernández I (see Sáenz-Santamaría et al). 2003; 47:337-340

Catalina-Fernández I (see Sáenz-Santamaría et al). 2003; 47:470-474

Catterall N (see Zardawi et al). 2003;47:704-705 (Letter)

Cavaco BM, Torrinha F, Mendonça E, Pratas S, Boavida J, Sobrinho LG, Leite V. Preoperative Diagnosis of Suspicious Parathyroid Adenomas by RT-PCR Using mRNA Extracted from Leftover Cells in a Needle Used for Ultrasoni-cally Guided Fine Needle Aspiration Cytology. 2003;47:5-12

Chaipipat M (see Tanboon et al). 2003;47:1143-1144 (Letter)

Chakka E (see Malle et al). 2003;47:129-134

Chan AB-W, Chan W-Y, Chow JH-S. Cytologic Features of NK/T-Cell Lymphoma. 2003;47:595-601

Chan AC-L, Chan JK-C, Yan KW, Kwong YL. Anaplastic Large Cell Lymphoma Presenting as a Pleural Effusion and Mimicking Primary Effusion Lymphoma: A Report of 2 Cases. 2003;47:809-816

Chan JK-C (see Chan et al). 2003;47:809-816

Chan W-Y (see Chan et al). 2003;47:595-601

Chang AR (see Yuan et al). 2003;47:415-420 
Chaturvedi S (see Arora et al). 2003;47:216-220

Chauvin S (see Yarkin et al). 2003;47:450-456

Chen KTK. Crush Cytology of Melanocytoma of the Spinal Cord: A Case Report. 2003;47:1091-1094

Chen KTK. Crush Cytology of Rosai-Dorfman Disease of the Central Nervous System: A Report of 2 Cases. 2003;47:1111-1115

Cheung LKN (see Ng et al). 2003;47:159-166

Cheung LKN (see Ng et al). 2003;47:56-64

Cheung LKN (see Ng et al). 2003;47:1063-1068

Cheung YK (see Gupta et al). 2003;47:393-398

Chiedozi LC (see El Hag et al). 2003;47:387-392

Chiu CSC (see Ng et al). 2003;47:373-380

Choi C (see Ko et al). 2003;47:727-732

Chow JCY (see Ng et al). 2003;47:373-380

Chow JCY (see Ng et al). 2003;47:56-64

Chow JH-S (see Chan et al). 2003;47:595-601

Chuah KL (see Goh and Chuah). 2003;47:293-298

Chuah KL (see Goh et al). 2003;47:65-70

Ciampolillo A (see Triggiani et al). 2003;47:1141-1143 (Letter)

Clark D (see Zardawi et al). 2003;47:1077-1081

Clark DP (see Allen et al). 2003;47:183-187

Clark DP (see Pu and Clark). 2003;47:247-252

Clark DP (see Segev et al). 2003;47:709-722

Clarke R (see Vesoulis et al). 2003;47:668-672

Cohen H (see Munichor et al). 2003;47:270-274

Cohen J-M (see Wiriosuparto et al). 2003;47:673-678

Collins BT (see Ponder and Collins). 2003;47:571-574

Córdova-Ramírez S (see Castro-Gómez et al). 2003;47: 590-594

Cubero J-M (see Lerma et al). 2003;47:575-580

Cuevas J (see Hardisson et al). 2003;47:259-264

Cytopathology Education and Technology Consortium. Daily Workload Guidelines for Cyto-technologists Utilizing Automated, Assisted-Screening Technologies. 2003;47:463-466

\section{D}

da Silva VD. Alternative, Cost-Effective Fungus-Staining Method for Control Slides in Cytology and Histopathology. 2003;47:1043-1044

Dankoff J (see Vesoulis et al). 2003;47:668-672

Das DK (see Pathan et al). 2003;47:299-303

Das K, Hameed M, Heller D, Mirani N, Doty N, Benevenia J, Patterson F, Aisner S. Liquid-Based vs. Conventional Smears in Fine Needle Aspiration of Bone and Soft Tissue Tumors. 2003;47:197-201

Daskalopoulou D (see Veneti et al). 2003;47:188-192

Dass A (see Mohan et al). 2003;47:792-794

Davis-Devine S, Day SJ, Freund GG. New Red Blood Cell Lysing Fixative for Use in Fine Needle Aspiration and Fluid Cytology. 2003;47:630-636
Dawar R (see Singh et al). 2003;47:739-743

Day SJ (see Davis-Devine et al). 2003;47:630-636

Dayer AM (see Basir et al). 2003;47:733-738

De Agustín PDA (see Vaillo Vinagre et al). 2003;47:485489

De Frias DVS (see Okonkwo et al). 2003;47:435-442

de la Fuente A (see Ortiz-Rey et al). 2003;47:490-494

DeFrias DVS (see Gong et al). 2003;47:1082-1086

DeFrias D (see Williamson et al). 2003;47:749-752

Dejmek A. Fine Needle Aspiration Cytology of an Ovarian Luteinized Follicular Cyst Mimicking a Granulosa Cell Tumor: A Case Report. 2003;47:1059-1062

DellaGiustina D, Falconieri G, Bonifacio-Gori D, Zanconati F, DiBonito L, Pizzolitto S. Bronchial Cytology in Pleural Mesothelioma: A Report of 3 Positive Cases, Including 1 Diagnosed Initially on Bronchial Brushings. 2003;47:1017-1022

Demirci SS (see Yang and Demirci). 2003;47:563-570

Dempsey PJ (see Madigan et al). 2003;47:783-786

Deshpande AH, Munshi MM. Fine Needle Capillary Sampling of Eyelid Masses: A Study of 70 Cases. 2003; 47:349-358

Destouni C (see Malle et al). 2003;47:129-134

Devi MG (see Madhumathi et al). 2003;47:101-103 (Letter)

Devi VL (see Madhumathi et al). 2003;47:101-103 (Letter)

Dey P (see Rajesh et al). 2003;47:177-182

Diaz A (see Yarkin et al). 2003;47:450-456

Diaz L (see Okonkwo et al). 2003;47:435-442

DiBonito L (see DellaGiustina et al). 2003;47:1017-1022

Divani S, Vardouli A, Exarhos N, Lioupis A. Endometriosis in the Differential Diagnosis of Abdominal Wall Masses. 2003;47:944-945 (Letter)

Dobeck S (see Melsheimer et al). 2003;47:124-128

Donnez J (see Weynand et al). 2003;47:149-153

Doty N (see Das et al). 2003;47:197-201

Doviner V (see Maly et al). 2003;47:1131-1134

Du M-Q (see El Hamidi et al). 2003;47:117-123

Duarte G (see Bettini et al). 2003;47:545-549

Duarte-Torres R (see Castro-Gómez et al). 2003;47:590-594

Duncan J (see Zardawi et al). 2003;47:704-705 (Letter)

Duncan J (see Zardawi and Duncan). 2003;47:1038-1042

Dundr P (see Glosová et al). 2003;47:1087-1090

Dušková J, Janotová D, Svobodová E, Novák Z, Tretiník P. Fine Needle Aspiration Biopsy of Mixed Medullary-Follicular Thyroid Carcinoma: A Report of Two Cases. 2003;47:71-77

\section{E}

Effler J (see Glosová et al). 2003;47:1087-1090

Ekalaksananan T (see Pientong et al). 2003;47:616-623

Ekinci C (see Soyuer et al). 2003;47:581-589

El Hag IA, Chiedozi LC, Al Reyees FA, Kollur SM. Fine Needle Aspiration Cytology of Head and Neck Mass- 
es: Seven Years' Experience in a Secondary Care Hospital. 2003;47:387-392

El Hag IA, Kollur SM. Fine Needle Aspiration Cytology of Pilomatrixoma of the Neck Region: Differentiation from Metastatic Undifferentiated Nasopharyngeal Carcinoma. 2003;47:526-528 (Letter)

El Hamidi A, Kocjan G, Du M-Q. Clonality Analysis of Archival Cervical Smears: Correla-tion of Monoclonality with Grade and Clinical Behavior of Cervical Intraepithelial Neoplasia. 2003;47:117-123

Elhosseiny A (see Zhang et al). 2003;47:645-648

El-Sahrigy D (see Zhang et al). 2003;47:645-648

Emoto K (see Tashiro et al). 2003;47:265-269

Emura I (see Ishida et al). 2003;47:1095-1098

Engh V (see Sauer et al). 2003;47:768-773

Erkıllı̧ S, Özsaraç C, Küllü S. Sputum Cytology for the Diagnosis of Lung Cancer: Comparison of Smear and Modified Cell Block Methods. 2003;47:1023-1027

Erozan YS (see Parwani et al). 2003;47:325-331

Ersöz C, Yağmur M, Ersöz TR, Yalaz M. Preoperative Brush and Impression Cytology in Ocular Surface Squamous Neoplasms. 2003;47:13-15

Ersöz TR (see Ersöz et al). 2003;47:13-15

Escanhoela C (see França et al). 2003;47:332-336

Escobar J (see Ribas et al). 2003;47:705-708 (Letter)

Eulalia E (see Lerma et al). 2003;47:575-580

Evren I (see Kabukcuoglu et al). 2003;47:311-313 (Letter)

Exarhos N (see Divani et al). 2003;47:944-945 (Letter)

\section{$\mathbf{F}$}

Fagnani F (see Bergeron and Fagnani). 2003;47:753-761

Falconieri G (see DellaGiustina et al). 2003;47:1017-1022

Fanning CV (see Caraway et al). 2003;47:777-782

Farinola MA, Lawler LP, Rosenthal D. Plasmacytoma with Involvement of the Urinary Bladder: Report of a Case Diagnosed by Urine Cytology. 2003;47:787-791

Fauck R (see Gupta et al). 2003;47:393-398

Fauck R (see Gupta et al). 2003;47:550-554

Fernández de Mera JJ (see Sáenz-Santamaría et al). 2003; 47:337-340

Fernández GC (see Ortiz-Rey et al). 2003;47:490-494

Fernández-Fernández I (see Pérez-Alonso and FernándezFernández). 2003;47:524-526 (Letter)

Fernandez-Mera JJ (see Sáenz-Santamaría et al). 2003;47:1-4

Fernández-Mera JJ (see Sáenz-Santamaría et al). 2003;47: $470-474$

Ferns S (see Kumar et al). 2003;47:528-529 (Letter)

Fleisher SR (see Nassar et al). 2003;47:762-767

Focchi J (see Mattosinho de Castro Ferraz et al). 2003;47: $154-158$

França AVC, Valério HMG, Trevisan M, Escanhoela C, Sevá-Pereira T, Zucoloto S, Martinelli A, Soares EC. Fine Needle Aspiration Biopsy for Improving the
Diagnostic Accuracy of Cut Needle Biopsy of Focal Liver Lesions. 2003;47:332-336

Francis IM (see Pathan et al). 2003;47:299-303

Fraser JL (see Ayata et al). 2003;47:973-978

Freund GG (see Davis-Devine et al). 2003;47:630-636

Fukuda M (see Mori et al). 2003;47:480-484

Furu I (see Sauer et al). 2003;47:768-773

Furuta N (see Satoh et al). 2003;47:1140-1141 (Letter)

Furuta T (see Kimura et al). 2003;47:1099-1102

\section{G}

Galant C (see Weynand et al). 2003;47:149-153

Galera H (see Lerma et al). 2003;47:575-580

Ganesan R (see Hejmadi et al). 2003;47:517-518 (Letter)

Gangane N (see Shivkumar et al). 2003;47:110-111 (Letter)

Gansler T (see Grossniklaus et al). 2003;47:239-246

Garbagnati F (see Kalogeraki et al). 2003;47:363-367

Garcia LW (see Ayata et al). 2003;47:973-978

Garcia-Asenjo JL (see Arias et al). 2003;47:1145-1146 (Letter)

García-Prats MD (see Vera-Alvarez et al). 2003;47:1107-1110

Gattuso P (see Lal et al). 2003;47:951-959

Gaur S (see Mehdi et al). 2003;47:529-531 (Letter)

Gayathri Devi M (see Premalata et al). 2003;47:475-479

Genç Y (see Soyuer et al). 2003;47:581-589

Geramizadeh B, Boob R, Talei AR, Rasekhi A. Fine Needle Aspiration Cytology in Hydatid Cyst of the Breast. 2003;47:701-702 (Letter)

Geramizadeh B, Hooshmand F, Kumar PV. Brush Cytology of Colorectal Malignancies. 2003;47:431-434

Ghaffar H, Parwani A, Rosenthal DL. Fine Needle Aspiration Cytology of Hepatic Metastasis from a Meningeal Hemangiopericytoma: A Case Report. 2003;47:281286

Ghio AJ, Alexis N. Siderophages in Sputum. 2003;47: 695-696 (Letter)

Ghosal N (see Anand et al). 2003;47:467-469

Ghosal N (see Kapila et al). 2003;47:555-562

Ghotekar LH (see Kumar et al). 2003;47:522-524 (Letter)

Gil-da Costa (see Barroca et al). 2003;47:1055-1058

Gill SS (see Kapila et al). 2003;47:555-562

Giovagnoli MR (see Pisani et al). 2003;47:520-522 (Letter)

Glosová L, Dundr P, Effler J, Růžičková M. Gallbladder Carcinoma Cells in Cerebrospinal Fluid as the First Manifestation of a Tumor: A Case Report. 2003; 47:1087-1090

Goel MM, Agarwal PK, Agarwal S. Primary Rosai-Dorfman Disease of Bone Without Lymphadenopathy Diagnosed by Fine Needle Aspiration Cytology: A Case Report. 2003;47:1119-1122

Goh SGN, Chuah KL, Tan PH, Tan NG. Role of FNAC in Metastasizing Malignant Mixed Tumor of the External Auditory Canal: A Case Report. 2003;47:65-70

Goh SGN, Chuah KL. Role of Intraoperative Smear 
Cytology in the Diagnosis of Anaplastic Oligodendroglioma: A Case Report. 2003;47:293-298

Gologan A (see Wiriosuparto et al). 2003;47:673-678

Gomez RS (see Guimarães et al). 2003;47:1074-1076

Gómez-Aracil V (see Mayayo et al). 2003;47:1116-1118

Gong G (see Jun et al). 2003;47:685-687

Gong Y, DeFrias DVS, Nayar R. Pitfalls in Fine Needle Aspiration Cytology of Extraadrenal Paraganglioma: A Report of 2 Cases. 2003;47:1082-1086

Gonzalez-Campora R (see Lerma et al). 2003;47:575-580

González-Peramato P (see Hardisson et al). 2003;47:259-264

Gonzalez L (see Arias et al). 2003;47:1145-1146 (Letter)

Gorlier C (see Oregioni et al). 2003;47:98-99 (Letter)

Goulart RA (see Cao et al). 2003;47:1033-1037

Granja NVM (see Longatto-Filho et al). 2003;47:317-318 (Letter)

Grossniklaus HE, Stulting RD, Gansler T, Aaberg TM Jr. Aspiration Cytology of the Conjunctival Surface. 2003;47:239-246

Gu M (see Zhang and Gu). 2003;47:253-258

Guimarães ALS, Perdigão PF, Siqueira FM, Castro WH, Gomez RS. Oral Metastasis of Breast Carcinoma Diagnosed by Fine Needle Aspiration Cytology: A Case Report. 2003;47:1074-1076

Günel Ö (see Topal et al). 2003;47:320-321 (Letter)

Gunn R (see Okonkwo et al). 2003;47:435-442

Gunn R (see Williamson et al). 2003;47:749-752

Gupta N (see Karak et al). 2003;47:227-238

Gupta R (see Anand et al). 2003;47:467-469

Gupta RK, Cheung YK, AlAnsari AG, Naran S, Lallu S, Fauck R. Value of Image-Guided Needle Aspiration Cytology in the Assessment of Pelvic and Retroperitoneal Masses: A Study of 112 Cases. 2003;47:393-398

Gupta RK, Naran S, Lallu S, Fauck R. Diagnostic Value of Needle Aspiration Cytology in the Assessment of Palpable Axillary Lymph Nodes: A Study of 336 Cases. 2003;47:550-554

Gupta S (see Jain et al). 2003;47:381-386

Gupta S, Sodhani P, Jain S. Acantholytic Cells Exfoliated from Pemphigus Vulgaris of the Uterine Cervix: A Case Report. 2003;47:795-798

Gupta S, Sodhani P, Jain S. Macroconidia of Fusarium Species: An Unusual Finding in Cervical Smears. 2003;47:41-44

Gupta SK (see Brahmi et al). 2003;47:443-449

Gutiérrez Martín A (see Vaillo Vinagre et al). 2003;47:485-489

H

Haack LA, Selvaggi SM. "Daisy" Cells in Peritoneal Washings. 2003;47:949-950 (Letter)

Haba T, Wakasa K, Sasaki M. Well-Differentiated Papillary Mesothelioma in the Pelvic Cavity: A Case Report. 2003;47:88-92
Hajdu SI. Acta Cytologica 40 Years Ago: Volume VII, Number 1, 1963. 2003;47:688-690

Hameed M (see Das et al). 2003;47:197-201

Han J (see Kim and Han). 2003;47:1103-1106

Han J (see Park et al). 2003;47:624-629

Han K-C (see Ng et al). 2003;47:373-380

Hanai N (see Okamura et al). 2003;47:209-215

Handa U (see Mohan et al). 2003;47:792-794

Harada H (see Kawahara et al). 2003;47:1127-1130

Hardisson D, González-Peramato P, Perna C, Cuevas J, Sánchez-Fernández de la Vega J. Fine Needle Aspiration Cytology of Solid Papillary Carcinoma of the Breast: A Report of Four Cases. 2003;47:259-264

Hasegawa Y, Ishida Y, Kato K, Ijiri R, Miyake T, Nishimata S, Watanabe T, Namba I, Hayabuchi Y, Kigasawa H, Tanaka Y. Pancreatoblastoma: A Case Report with Special Emphasis on Squa-moid Corpuscles with Optically Clear Nuclei Rich in Biotin. 2003;47:679-684

Hashimoto S (see Kimura et al). 2003;47:1099-1102

Haumont E (see Weynand et al). 2003;47:149-153

Hauptman E (see Yarkin et al). 2003;47:450-456

Hayabuchi Y (see Hasegawa et al). 2003;47:679-684

Hecht JL (see Qian and Hecht). 2003;47:723-726

Heim M (see Sauer et al). 2003;47:768-773

Hejmadi R, Ganesan R, Kamal NG. Malignant Transformation of a Well-Differentiated Peritoneal Papillary Mesothelioma. 2003;47:517-518 (Letter)

Heller D (see Das et al). 2003;47:197-201

Hemachandran M (see Nijhawan et al). 2003;47:193-196

Henry MR (see Studeman et al). 2003;47:605-610

Hiatt RA (see Kinney et al). 2003;47:167-171

Higaki T (see Kobayashi et al). 2003;47:103-106 (Letter)

Hira PR (see Pathan et al). 2003;47:299-303

Hirokawa M (see Kuma et al). 2003;47:399-404

Hirokawa M (see Sakaki et al). 2003;47:321-324 (Letter)

Hirokawa M (see Tashiro et al). 2003;47:265-269

Hoebling W (see Pokieser et al). 2003;47:108-110 (Letter)

Holck AM (see Sauer et al). 2003;47:768-773

Honma S (see Sasagawa et al). 2003;47:410-414

Hooshmand F (see Geramizadeh et al). 2003;47:431-434

Horiuchi F (see Nagashima et al). 2003;47:1028-1032

Hoshi N (see Watanabe et al). 2003;47:99-101 (Letter)

Hoshiai H (see Watanabe et al). 2003;47:78-82

Huang R, Jaffer S. Imprint Cytology of Metastatic Sialoblastoma: A Case Report. 2003;47:1123-1126

Hurtado-López LM (see Castro-Gómez et al). 2003;47: 590-594

Hurtl I (see Pokieser et al). 2003;47:108-110 (Letter)

\section{I}

Iancu TC (see Munichor et al). 2003;47:270-274

Ijiri R (see Hasegawa et al). 2003;47:679-684

Imamura Y (see Mori et al). 2003;47:480-484 
Ioannidou-Mouzaka L (see Veneti et al). 2003;47:188-192 Ioffe OB (see Studeman et al). 2003;47:605-610

Ishibashi Y (see Kuwabara et al). 2003;47:515-517 (Letter) Ishida GM, Motoyama T, Watanabe T, Emura I. Clear Cell Carcinoma Arising in a Cesarean Section Scar: Report of a Case with Fine Needle Aspiration Cytology. 2003;47:1095-1098

Ishida Y (see Hasegawa et al). 2003;47:679-684

Ishihara T (see Okamura et al). 2003;47:209-215

Ishikawa Y (see Satoh et al). 2003;47:1140-1141 (Letter)

Iuchi K (see Tashiro et al). 2003;47:265-269

\section{$\sqrt{2}$}

Jaffer S (see Huang and Jaffer). 2003;47:1123-1126 Jahadi SHR (see Mehriyar et al). 2003;47:531-533 (Letter) Jain P (see Anand et al). 2003;47:467-469

Jain S (see Gupta et al). 2003;47:41-44

Jain S (see Gupta et al). 2003;47:795-798

Jain S (see Khurana and Jain). 2003;47:946-948 (Letter) Jain S, Gupta S, Kumar N, Sodhani P. Extracellular Hyaline Material in Association with Other Cytologic Features in Aspirates from Collagenous Spherulosis and Adenoid Cystic Carcinoma of the Breast. 2003;47:381-386

Jang J (see Jun et al). 2003;47:685-687

Janotová D (see Dušková et al). 2003;47:71-77

Jara Facundo D, Quinonez G, Ravinsky E. Transmission Electron Microscopy of Fine Needle Aspiration Biopsies of Metastases: Accuracy of Both Techniques as Established by Biopsy Diagnoses. 2003;47:457-462 Jatiya L (see Kumar et al). 2003;47:528-529 (Letter) Jayrajpuri ZS (see Sherwani et al). 2003;47:506-508 Jhu I-K (see Ko et al). 2003;47:727-732

Jiménez-Heffernan JA, Sanz E, López-Ferrer P. Cytologic

Features of Subependymoma. 2003;47:319-320 (Letter) Jobo T, Arai T, Sato R, Kuramoto H. Clinicopathologic Relevance of Asymptomatic Endometrial Carcinoma. 2003;47:611-615

Joshi K (see Brahmi et al). 2003;47:443-449

Joshi K (see Nijhawan et al). 2003;47:193-196

Joshi K (see Rajesh et al). 2003;47:177-182

Jou C (see Ribas et al). 2003;47:705-708 (Letter)

Juhng S-W (see Ko et al). 2003;47:727-732

Jun S-Y, Jang J, Ahn S-H, Park JM, Gong G. Paragonimiasis of the Breast: Report of a Case Diagnosed by Fine Needle Aspiration. 2003;47:685-687

\section{K}

Kabukcuoglu F, Sungun A, Polat N, Evren I, Kabukcuoglu Y. Fine Needle Aspiration Cytology of Merkel Cell Carcinoma. 2003;47:311-313 (Letter)
Kabukcuoglu Y (see Kabukcuoglu et al). 2003;47:311-313 (Letter)

Kage M (see Kawahara et al). 2003;47:1127-1130

Kakudo K (see Kuma et al). 2003;47:399-404

Kalogeraki A, Garbagnati F, Santinami M, Zoras O. Ecadherin Expression on Fine Needle Aspiration Biopsies of Breast Invasive Ductal Carci-nomas and Its Relationship to Clinicopathologic Factors.2003;47:363367

Kamal NG (see Hejmadi et al). 2003;47:517-518 (Letter)

Kamei T (see Okamura et al). 2003;47:209-215

Kaneko C (see Kobayashi et al). 2003;47:1069-1073

Kapila K (see Singh et al). 2003;47:739-743

Kapila K (see Verma and Kapila). 2003;47:221-226

Kapila K, Ghosal N, Gill SS, Verma K. Cytomorphology of Lipomatous Tumors of Soft Tissue. 2003;47:555-562

Kapila K, Verma K. Cytology of Nipple Discharge in Florid Gynecomastia. 2003;47:36-40

Kapila K, Verma K. Fine Needle Aspiration Cytology of Epidermal Inclusion Cysts in the Male Breast. 2003;47: 315-317 (Letter)

Karak AK, Marwaha RK, Tandon N, Gupta N, Kochupillai N, Verma K. Cytologic Characterization of Postiodization Residual Goiter in Schoolchildren by Fine Needle Biopsy. 2003;47:227-238

Katayama S (see Kuma et al). 2003;47:399-404

Kato K (see Hasegawa et al). 2003;47:679-684

Katsumori T (see Kobayashi et al). 2003;47:309-311 (Letter)

Kaul S (see Melsheimer et al). 2003;47:124-128

Kavian N (see Mehriyar et al). 2003;47:531-533 (Letter)

Kawahara A, Harada H, Yokoyama T, Kage M. Cytomorphologic Features of a Polymorphous Low Grade Adenocarcinoma of the Palate: A Report of 2 Cases with Immunocytochemistry. 2003;47:1127-1130

Kay PA, Nascimento AG, Unni KK, Salomão DR. Chordoma: Cytomorphologic Findings in 14 Cases Diagnosed by Fine Needle Aspiration. 2003;47:202-208

Kaya M (see Soyuer et al). 2003;47:581-589

Kearney KA (see Kinney et al). 2003;47:167-171

Kecelj P (see Kern et al). 2003;47:426-430

Kern I, Kecelj P, Kos̆nik M, Mermolja M. Multinucleated Giant Cells in Bronchoalveolar Lavage. 2003;47:426430

Kerner H (see Munichor et al). 2003;47:270-274

Khanna G (see Sharma et al). 2003;47:359-362

Khurana N, Jain S. Granulocytic Sarcoma Presenting as a Breast Mass. 2003;47:946-948 (Letter)

Kigasawa H (see Hasegawa et al). 2003;47:679-684

Kikuchi I, Anbo J, Nakamura S-i, Sugai T, Sasou S, Yamamoto M, Oda Y, Shiratsuchi H, Tsuneyoshi M. Synovial Sarcoma of the Thyroid: Report of a Case with Aspiration Cytology Findings and Gene Analysis. 2003;47:495-500

Kim B (see Afify et al). 2003;47:341-348

Kim NR, Han J. Primary Giant Cell Tumor of Soft Tis- 
sue: Report of a Case with Fine Needle Aspiration Cytologic and Histologic Findings. 2003;47:1103-1106

Kimura J, Okamoto H, Yamamoto H, Mazaki T, Kitamura T. Cytologic Features of Atypical Polypoid Adenomyoma of the Endometrium: A Case Report. 2003;47: 287-292

Kimura M, Furuta T, Hashimoto S, Takano T, Nagao K. Oncocytic Carcinoma of the Parotid Gland: A Case Report. 2003;47:1099-1102

Kini H, Bhat S. Cardiac Tamponade: An Unusual Presentation of Metastatic Squamous Cell Carcinoma of the Larynx. 2003;47:113-114 (Letter)

Kinjo M, Yohena C, Kunishima N. Intracytoplasmic Lumina in Medullary Carcinoma of the Thyroid Gland: Report of a Case with Cytologic and Immunocytochemical Features. 2003;47:663-667

Kinney W, Sawaya GF, Sung H-Y, Kearney KA, Miller M, Hiatt RA. Stage at Diagnosis and Mortality in Patients with Adenocarcinoma and Adenosquamous Carcinoma of the Uterine Cervix Diagnosed as a Consequence of Cytologic Screening. 2003;47:167-171

Kishore S (see Shivkumar et al). 2003;47:110-111 (Letter)

Kitamura T (see Kimura et al). 2003;47:287-292

Knop N (see Wen et al). 2003;47:135-140

Ko H-M, Jhu I-K, Yang S-H, Lee J-H, Nam J-H, Juhng SW, Choi C. Clinicopathologic Analysis of Fine Needle Aspiration Cytology of the Thyroid: A Review of 1,613 Cases and Correla-tion with Histopathologic Diagnoses. 2003;47:727-732

Kobayashi TK (see Okuyama et al). 2003;47:696-698 (Letter)

Kobayashi TK, Moritani S, Katsumori T, Urabe M. Cytologic Features of Vaginal Discharge Obtained After Uterine Artery Embolization for Uterine Leiomyomata. 2003;47:309-311 (Letter)

Kobayashi TK, Ueda M, Nishino T, Moritani S, Higaki T, Bamba M. Cytologic Detection of Cryptococcosis Coexisting with Herpes Simplex Virus Infection in Sputum: Use of Liquid-Based, Thin-Layer Preparations. 2003;47:103-106 (Letter)

Kobayashi TK, Ueda M, Nishino T, Muramatsu M, Moritani S, Seki H, Araki H, Kaneko C. Papillary Clusters as a Diagnostic Pitfall in Urinary Cytology of Pseudosarcomatous Fibromyxoid Tumor of the Bladder: A Case Report. 2003;47:1069-1073

Kochupillai N (see Karak et al). 2003;47:227-238

Kocjan G (see El Hamidi et al). 2003;47:117-123

Koda K (see Nagashima et al). 2003;47:1028-1032

Kodama S (see Sasagawa et al). 2003;47:410-414

Koivurinne KI, Shield PW. Thin-Layer Preparations of Dithiothreitol-Treated Bronchial Washing Specimens. 2003;47:637-644

Kollur SM (see El Hag and Kollur). 2003;47:526-528 (Letter)

Kollur SM (see El Hag et al). 2003;47:387-392

Komal HS (see Brahmi et al). 2003;47:443-449
Komorowski RA (see Basir et al). 2003;47:733-738

Kong JHB (see Ng and Kong). 2003;47:27-35

Kong JHB (see Ng et al). 2003;47:698-701 (Letter)

Kongyingyoes B (see Pientong et al). 2003;47:616-623

Konomi N (see Yarkin et al). 2003;47:450-456

Kos̆nik M (see Kern et al). 2003;47:426-430

Koss LG (see Payandeh and Koss). 2003;47:421-425

Kotwal SA (see Mohan et al). 2003;47:792-794

Krassilnik N (see Wiriosuparto et al). 2003;47:673-678

Krebs P (see Cao et al). 2003;47:1033-1037

Krishnamurthy S (see Madigan et al). 2003;47:783-786

Krishnamurthy S (see Tabatabai and Krishnamurthy). 2003;47:106-107 (Letter)

Kritpetcharat O (see Pientong et al). 2003;47:616-623

Küllü S (see Erkılıç et al). 2003;47:1023-1027

Kuma S, Hirokawa M, Miyauchi A, Kakudo K, Katayama S. Cytologic Features of Hyalinizing Trabecular Adenoma of the Thyroid. 2003;47:399-404

Kumar N (see Jain et al). 2003;47:381-386

Kumar PV (see Geramizadeh et al). 2003;47:431-434

Kumar PV (see Mehriyar et al). 2003;47:531-533 (Letter)

Kumar PV (see Monabati et al). 2003;47:702-704 (Letter)

Kumar R (see Anand et al). 2003;47:467-469

Kumar S, Ferns S, Jatiya L. A Rare Case of Cryptococcosis Diagnosed by Fine Needle Aspiration Cytology. 2003;47:528-529 (Letter)

Kumar S, Ghotekar LH, Thappa DM, Smile R. Diagnosis of Chondroid Syringoma by Fine Needle Aspiration Cytology. 2003;47:522-524 (Letter)

Kunishima N (see Kinjo et al). 2003;47:663-667

Kuramoto H (see Jobo et al). 2003;47:611-615

Kuwabara H, Mori H, Uda H, Takei K, Ishibashi Y, Takatani N. Nodal Neuroendocrine (Merkel Cell) Carcinoma Without an Identifiable Primary Tumor. 2003;47:515-517 (Letter)

Kwong YL (see Chan et al). 2003;47:809-816

$\mathbf{L}$

Laforga JB. Cytologic Imprints in Intraoperative Consultations for Gastric Heterotopic Pancreas. 2003;47: 945-946 (Letter)

Lal A (see Okonkwo et al). 2003;47:435-442

Lal A, Ariga R, Gattuso P, Nemcek AA, Nayar R. Splenic Fine Needle Aspiration and Core Biopsy: A Review of 49 Cases. 2003;47:951-959

Lallu S (see Gupta et al). 2003;47:393-398

Lallu S (see Gupta et al). 2003;47:550-554

Larson MA (see Rowe et al). 2003;47:602-604

Lawler LP (see Farinola et al). 2003;47:787-791

Le Fichoux Y (see Oregioni et al). 2003;47:98-99 (Letter)

Lee CYK (see $\mathrm{Ng}$ et al). 2003;47:1063-1068

Lee J-H (see Ko et al). 2003;47:727-732

Leite V (see Cavaco et al). 2003;47:5-12 
Lepore M (see Zeppa et al). 2003;47:501-505

Lerma E, Arguelles R, Rigla M, Otal C, Cubero J-M, Bagué S, Carreras AM, Eulalia E, Gonzalez-Campora R, Galera H, Prat J. Comparative Findings of Lymphocytic Thyroiditis and Thyroid Lymphoma. 2003; 47:575-580

Leung SW (see Bédard et al). 2003;47:979-984

Li ASM (see Ng et al). 2003;47:56-64

Li ASM (see Ng et al). 2003;47:159-166

Li ASM (see Ng et al). 2003;47:1063-1068

Li L (see Pan et al). 2003;47:45-50

Lioupis A (see Divani et al). 2003;47:944-945 (Letter)

LiVolsi V (see Nassar et al). 2003;47:313-315 (Letter)

Logroño R (see Tibbs et al). 2003;47:172-176

Lombardo V (see Longatto-Filho et al). 2003;47:317-318 (Letter)

Longatto-Filho A, Bisi H, Bortolan J, Granja NVM, Lombardo V. Cytologic Diagnosis of Metastatic Sarcoma in Effusions. 2003;47:317-318 (Letter)

Loo CKC, Burchett IJ. Fine Needle Aspiration Biopsy of Neuroendocrine Breast Carcinoma Metastatic to the Thyroid: A Case Report. 2003;47:83-87

López-Ferrer P (see Jiménez-Heffernan et al). 2003;47: 319-320 (Letter)

López-López JI (see Vera-Alvarez et al). 2003;47:11071110

Lorenzana RR (see Cao et al). 2003;47:1033-1037

Lu DW, Pirog EC, Zhu X, Wang HL, Pinto KR. Prevalence and Typing of HPV DNA in Atypical Squamous Cells in Pregnant Women. 2003;47:1008-1016

Lu Y (see Vesoulis et al). 2003;47:668-672

\section{$\mathbf{M}$}

Madhumathi DS, Sundareshan TS, Amirtham U, Devi VL, Devi MG. Blastic Extramedullary Myeloid Cell Tumor of the Lymph Node Diagnosed by Fine Needle Aspiration. 2003;47:101-103 (Letter)

Madigan MN, Dempsey PJ, Krishnamurthy S. Ultrasound-Guided Fine Needle Aspiration Cytodiagnosis of Leiomyosarcoma Metastatic to the Breast: A Case Report. 2003;47:783-786

Maeda S (see Okuyama et al). 2003;47:696-698 (Letter)

Maegawa H (see Mori et al). 2003;47:480-484

Magdalena CJ (see Ortiz-Rey et al). 2003;47:490-494

Maheshwari V (see Mehdi et al). 2003;47:529-531 (Letter)

Maiorano E (see Triggiani et al). 2003;47:1141-1143 (Letter)

Malik A (see Singh et al). 2003;47:368-372

Malle D, Pateinakis P, Chakka E, Destouni C. Experience with a Thin-Layer, Liquid-Based Cervical Cytologic Screening Method. 2003;47:129-134

Mallik MK (see Pathan et al). 2003;47:299-303

Maly A (see Maly et al). 2003;47:1131-1134
Maly B, Maly A, Doviner V, Reinhartz T, Sherman Y. Fine Needle Aspiration Biopsy of Intraparotid Schwannoma: A Case Report. 2003;47:1131-1134

Mansbacher S (see Wen et al). 2003;47:135-140

Manucha V (see Mishra et al). 2003;47:1001-1007

Marconi S (see Cao et al). 2003;47:1033-1037

Marigil-Gómez M (see Vera-Alvarez et al). 2003;47:11071110

Marshall CJ (see Rowe et al). 2003;47:602-604

Martinelli A (see França et al). 2003;47:332-336

Martinez S (see Mayayo et al). 2003;47:1116-1118

Marty P (see Oregioni et al). 2003;47:98-99 (Letter)

Marwaha RK (see Karak et al). 2003;47:227-238

Masih VF (see Singh et al). 2003;47:960-964

Massart F (see Weynand et al). 2003;47:149-153

Mattosinho de Castro Ferraz MDG, Focchi J, Stavale JN, Nicolau SM, Rodrigues de Lima G, Baracat EC. Atypical Glandular Cells of Undetermined Significance: Cytologic Predictive Value for Glandular Involvement in High Grade Squamous Intraepithelial Lesions. 2003;47:154-158

Mayayo E, Blázquez S, Gómez-Aracil V, Saurí A, Martinez S. Spleen Metastasis from Thyroid Carcinoma: Report of a Case with Diagnosis by Fine Needle Aspiration Cytology. 2003;47:1116-1118

Mazaki T (see Kimura et al). 2003;47:287-292

Mehdi G, Maheshwari V, Gaur S, Sharma R. Histoid Leprosy: Diagnosis By Fine Needle Aspiration Cytology. 2003;47:529-531 (Letter)

Mehriyar M, Jahadi SHR, Kavian N, Kumar PV. Testicular Germ Cell Tumor Metastatic to the Retina: Vitreous Fluid Cytologic Findings. 2003;47:531-533 (Letter)

Melamed MR (see Zhang et al). 2003;47:645-648

Melsheimer P, Kaul S, Dobeck S, Bastert G. Immunocytochemical Detection of HPV High-Risk Type L1 Capsid Proteins in LSIL and HSIL as Compared with Detection of HPV L1 DNA. 2003;47:124-128

Mena M (see Omodeo-Zorini et al). 2003;47:985-990

Mendoza ML (see Arias et al). 2003;47:1145-1146 (Letter)

Mendonça E (see Cavaco et al). 2003;47:5-12

Mermolja M (see Kern et al). 2003;47:426-430

Miller M (see Kinney et al). 2003;47:167-171

Mirani N (see Das et al). 2003;47:197-201

Mishra K (see Sharma et al). 2003;47:359-362

Mishra K, Manucha V, Sengupta R, Singh K, Aggarwal N. Cytomorphology and Histomorphology After Thermal Endometrial Ablation: Early Changes. 2003;47:1001-1007

Miyake T (see Hasegawa et al). 2003;47:679-684

Miyauchi A (see Kuma et al). 2003;47:399-404

Miyazaki M (see Nagashima et al). 2003;47:1028-1032

Mo R (see Yarkin et al). 2003;47:450-456

Mohan H, Handa U, Amanjit, Kotwal SA, Dass A. Adenoid Cystic Carcinoma of the External Auditory Canal: A Case Report with Diagnosis by Fine Needle Aspiration. 2003;47:792-794 
Monabati A, Kumar PV, Roozbehi H, Torabinezhad S. Cytologic Findings in Metastatic Oligodendroglioma. 2003;47:702-704 (Letter)

Monga G (see Omodeo-Zorini et al). 2003;47:985-990

Monobe Y (see Tashiro et al). 2003;47:265-269

Mori H (see Kuwabara et al). 2003;47:515-517 (Letter)

Mori M, Imamura Y, Maegawa H, Yoshida H, Naiki H, Fukuda M. Cytology of Pleural Effusion Associated with Disseminated Infection Caused by VaricellaZoster Virus in an Immunocompromised Patient: A Case Report. 2003;47:480-484

Moritani S (see Kobayashi et al). 2003;47:103-106 (Letter)

Moritani S (see Kobayashi et al). 2003;47:309-311 (Letter)

Moritani S (see Kobayashi et al). 2003;47:1069-1073

Motoyama T (see Ishida et al). 2003;47:1095-1098

Mourad WA, Nazer MA, Tulbah A. Cytomorphologic Differentiation of Hodgkin's Lymphoma and Ki-1+ Anaplastic Large Cell Lymphoma in Fine Needle Aspirates. 2003;47:744-748

Mukherjee G (see Premalata et al). 2003;47:475-479

Munichor M, Cohen H, Volpin G, Kerner H, Iancu TC. Chromium-Induced Lymph Node Histiocytic Proliferation After Hip Replacement: A Case Report. 2003; 47:270-274

Munshi MM (see Deshpande and Munshi). 2003;47:349_ 358

Muramatsu M (see Kobayashi et al). 2003;47:1069-1073

\section{$\mathbf{N}$}

Nagao K (see Kimura et al). 2003;47:1099-1102

Nagashima T, Suzuki M, Yagata H, Nikaido T, Horiuchi F, Koda K, Miyazaki M. Intraoperative Cytologic Diagnosis of Sentinel Node Metastases in Breast Cancer. 2003;47:1028-1032

Naiki H (see Mori et al). 2003;47:480-484

Nakai H (see Watanabe et al). 2003;47:78-82

Nakamura S-i (see Kikuchi et al). 2003;47:495-500

Nam J-H (see Ko et al). 2003;47:727-732

Namba I (see Hasegawa et al). 2003;47:679-684

Naran S (see Gupta et al). 2003;47:393-398

Naran S (see Gupta et al). 2003;47:550-554

Nascimento AG (see Kay et al). 2003;47:202-208

Nassar A, Fleisher SR, Nasuti JF. Value of Histiocyte Detection in Pap Smears for Predicting Endometrial Pathology: An Institutional Experience. 2003;47:762767

Nassar A, Saqi A, Baloch Z, LiVolsi V. Carcinoma Showing Thymuslike Element of the Thyroid. 2003;47:313315 (Letter)

Nasser I (see Pantanowitz et al). 2003;47:1050-1054

Nasuti JF (see Nassar et al). 2003;47:762-767

Nayar R (see Gong et al). 2003;47:1082-1086

Nayar R (see Lal et al). 2003;47:951-959
Nayar R (see Okonkwo et al). 2003;47:435-442

Nayar R (see Williamson et al). 2003;47:749-752

Nazer MA (see Mourad et al). 2003;47:744-748

Nelson R (see Vesoulis et al). 2003;47:668-672

Nemcek AA (see Lal et al). 2003;47:951-959

Neuhold N (see Pokieser et al). 2003;47:108-110 (Letter)

$\mathrm{Ng}$ HK (see Yuan et al). 2003;47:415-420

Ng W-K, Cheung LKN, Li ASM, Tse SKM, Pang S-W, Chow JCY. Thin-Layer Cytology Findings of Small Cell Carcinoma of the Lower Female Genital Tract: Review of Three Cases with Molecular Analysis. 2003; 47:56-64

Ng W-K, Cheung LKN, Li ASM. Warty (Condylomatous) Carcinoma of the Cervix: A Review of 3 Cases with Emphasis on Thin-Layer Cytology and Molecular Analysis for HPV. 2003;47:159-166

Ng W-K, Chiu CSC, Han K-C, Chow JCY. Mammary Pseudoangiomatous Stromal Hyperplasia: A Reappraisal of the Fine Needle Aspiration Cytology Findings. 2003;47:373-380

Ng W-K, Kong JHB, Wong WWC. Atypical Apocrine Metaplasia: A Diagnostic Pitfall in Fine Needle Aspiration Cytology of the Breast. 2003;47:698-701 (Letter)

$\mathrm{Ng} \mathrm{W-K,} \mathrm{Kong} \mathrm{JHB.} \mathrm{Significance} \mathrm{of} \mathrm{Squamous} \mathrm{Cells} \mathrm{in}$ Fine Needle Aspiration Cytology of the Breast: A Review of Cases in a Seven-Year Period.2003;47:27-35

Ng W-K, Lee CYK, Li ASM, Cheung LKN. Nodal Presentation of Nasal-Type NK/T-Cell Lymphoma: Report of Two Cases with Fine Needle Aspiration Cytology Findings. 2003;47:1063-1068

$\mathrm{Ng}$ W-K. Mammary Mucinous Carcinoma with Marked Cytoplasmic Hyalinization: A Report of 2 Cases with Emphasis on Fine Needle Aspiration Cytologic Findings. 2003;47:1045-1049

Ng W-K, Yip WW-L. Fine Needle Aspiration Cytology Findings of Cystic Hypersecretory Ductal Carcinoma of the Breast: A Reappraisal. 2003;47:513-515 (Letter)

Ng W-K. Thin-Layer (Liquid-Based) Cytologic Findings of Papillary Squamotransitional Cell Carcinoma of the Cervix: Review of Cases Over a 4-Year Period with Emphasis on Potential Diagnostic Pitfalls. 2003;47: 141-148

Ng W-K. Thin-Layer Cytology Findings of Papillary Adenosquamous Carcinoma of the Cervix: Report of a Case with Histologic Correlation and Molecular Analysis. 2003;47:649-656

Nicolau SM (see Mattosinho de Castro Ferraz et al). 2003; 47:154-158

Nijhawan R, Hemachandran M, Joshi K. Apoptosis in Breast Cancer. 2003;47:193-196

Nikaido T (see Nagashima et al). 2003;47:1028-1032

Nilkund J (see Basu and Nilkund). 2003;47:774-776

Nilsson B (see Wee and Nilsson). 2003;47:16-26

Nishimata S (see Hasegawa et al). 2003;47:679-684

Nishino K (see Sasagawa et al). 2003;47:410-414

Nishino T (see Kobayashi et al). 2003;47:103-106 (Letter) 
Nishino T (see Kobayashi et al). 2003;47:1069-1073

Noda K (see Watanabe et al). 2003;47:78-82

Novák Z (see Dušková et al). 2003;47:71-77

Nygård JF, Sauer T, Skjeldestad FE, Skare GB, Thoresen SØ. CIN 2/3 and Cervical Cancer After an ASCUS Pap Smear: A 7-Year, Prospective Study of the Norwegian Population-Based, Coordinated Screening Program. 2003;47:991-1000

\section{$\mathbf{O}$}

O'Malley FP (see Bédard et al). 2003;47:979-984

Oda Y (see Kikuchi et al). 2003;47:495-500

Ogura G (see Watanabe et al). 2003;47:99-101 (Letter)

Okamoto H (see Kimura et al). 2003;47:287-292

Okamura H, Kamei T, Sakuma N, Hanai N, Ishihara T. Ribonucleotide Reductase Immunoreactivity in Adenocarcinoma Cells and Malignant or Reactive Mesothelial Cells in Serous Effusions. 2003;47:209-215

Okonkwo AM, De Frias DVS, Gunn R, Diaz L, Schindler S, Lal A, Nayar R. Reclassification of "Atypical" Diagnoses in Endoscopic Retrograde Cholangiopancreaticography-Guided Biliary Brushings. 2003;47: 435-442

Okuyama T, Maeda S, Kobayashi TK, Takahashi R. Detection of Trichomonas vaginalis by Combined A6p and P65 Sequences using PCR Amplification from Papanicolaou-Stained Smears. 2003;47:696-698 (Letter)

Olivi A (see Parwani et al). 2003;47:325-331

Omodeo-Zorini E, Boldorini R, Viganò P, Mena M, Benigni E, Andorno S, Monga G. Sequence Analysis of the JC Virus Transcriptional Control Region Detected in Urine from HIV-Positive Patients. 2003;47:985990

Oregioni O, Marty P, Le Fichoux Y, Angeli K, Gorlier C, Rosenthal E, Perrin C. Cytology of Peritoneal Leishmaniasis in a Patient with AIDS. 2003;47:98-99 (Letter)

Ortiz-Rey JA, Fernández GC, Magdalena CJ, Álvarez C, Antón I, San Miguel P, de la Fuente A. Fine Needle Aspiration Appearance of Extragastrointestinal Stromal Tumor: A Case Report. 2003;47:490-494

Otal C (see Lerma et al). 2003;47:575-580

Özsaraç C (see Erkılıç et al). 2003;47:1023-1027

\section{$\mathbf{P}$}

Palombini L (see Zeppa et al). 2003;47:501-505

Pan Q, Belinson JL, Li L, Pretorius RG, Qiao YL, Zhang WH, Zhang X, Wu LY, Rong SD, Sun YT. A ThinLayer, Liquid-Based Pap Test for Mass Screening in an Area of China with a High Incidence of Cervical Carcinoma: A Cross-Sectional, Comparative Study. 2003;47:45-50

Panetti C (see Cao et al). 2003;47:1033-1037

Pang S-W (see Ng et al). 2003;47:56-64

Pantanowitz L, Upton MP, Wang HH, Nasser I. Cytomorphology of Verrucous Carcinoma of the Cervix: A Case Report. 2003;47:1050-1054

Panwar A (see Singh et al). 2003;47:960-964

Papasotiriou E (see Veneti et al). 2003;47:188-192

Park JM (see Jun et al). 2003;47:685-687

Park J-Y, Suh Y-L, Han J. Dysembryoplastic Neuroepithelial Tumor: Features Distinguishing It from Oligodendroglioma on Cytologic Squash Preparations. 2003;47:624-629

Parwani A (see Ghaffar et al). 2003;47:281-286

Parwani AV (see Allen et al). 2003;47:183-187

Parwani AV, Taylor DC, Burger PC, Erozan YS, Olivi A, Ali SZ. Keratinized Squamous Cells in Fine Needle Aspiration of the Brain: Cytopathologic Correlates and Differential Diagnosis. 2003;47:325-331

Pateinakis P (see Malle et al). 2003;47:129-134

Pathan SK, Francis IM, Das DK, Mallik MK, Sheikh ZA, Hira PR. Fine Needle Aspiration Cytologic Diagnosis of Toxoplasma Lymphadenitis: A Case Report with Detection of a Toxoplasma Bradycyst in a PapanicolaouStained Smear. 2003;47:299-303

Patterson F (see Das et al). 2003;47:197-201

Payandeh F, Koss LG. Nuclear Grooves in Normal and Abnormal Cervical Smears. 2003;47:421-425

Pello N (see Basir et al). 2003;47:733-738

Perdigão PF (see Guimarães et al). 2003;47:1074-1076

Pérez Barrios A (see Vaillo Vinagre et al). 2003;47:485489

Pérez-Alonso P, Fernández-Fernández I. Fine Needle Aspiration Cytology of a Cutaneous Bronchogenic Cyst. 2003;47:524-526 (Letter)

Perez-Ruiz J (see Vera-Alvarez et al). 2003;47:1107-1110

Perna C (see Hardisson et al). 2003;47:259-264

Perrin C (see Oregioni et al). 2003;47:98-99 (Letter)

Pientong C, Ekalaksananan T, Swadpanich U, Kongyingyoes B, Kritpetcharat O, Yuenyao P, Ruckait N. Immunocytochemical Detection of p16INK4a Protein in Scraped Cervical Cells. 2003;47:616-623

Pinto KR (see Lu et al). 2003;47:1008-1016

Pirog EC (see Lu et al). 2003;47:1008-1016

Pisani T, Vecchione A, Giovagnoli MR. Immunocytochemical Analysis of Fine Needle Aspiration Cytology to Improve Surgical Strategy in Thyroid Neoplasms. 2003;47:520-522 (Letter)

Pizzolitto S (see DellaGiustina et al). 2003;47:1017-1022

Pokieser W, Ulrich W, Neuhold N, Hoebling W, Hurtl I. Giant Cells in Poorly Differentiated (Insular) Carcinoma of the Thyroid. 2003;47:108-110 (Letter)

Polat N (see Kabukcuoglu et al). 2003;47:311-313 (Letter)

Pollett AF (see Bédard et al). 2003;47:979-984 
Ponder TB, Collins BT. Fine Needle Aspiration Biopsy of Gastric Duplication Cysts with Endoscopic Ultrasound Guidance. 2003;47:571-574

Pourvoyeur A (see Weynand et al). 2003;47:149-153

Prabhakaran PS (see Premalata et al). 2003;47:475-479

Prasoon D. Tuberculosis of the Intercostal Lymph Nodes. 2003;47:51-55

Prat J (see Lerma et al). 2003;47:575-580

Prats D (see Arias et al). 2003;47:1145-1146 (Letter)

Pratas S (see Cavaco et al). 2003;47:5-12

Premalata CS, Gayathri Devi M, Biswas S, Mukherjee G, Balu S, Sundareshan TS, Prabhakaran PS. Primitive Neuroectodermal Tumor of the Kidney: A Report of Two Cases Diagnosed by Fine Needle Aspiration Cytology. 2003;47:475-479

Pretorius RG (see Pan et al). 2003;47:45-50

$\mathrm{Pu}$ RT, Clark DP. Detection of DNA Methylation: Potential Applications to Diagnostic Cytopathology. 2003; 47:247-252

Puszkiewicz J (see Studeman et al). 2003;47:605-610

Q

Qian X, Hecht JL. Pancreatic Fine Needle Aspiration: A Comparison of Computed Tomographic and Endoscopic Ultrasonographic Guidance. 2003;47:723-726

Qiao YL (see Pan et al). 2003;47:45-50

Quinonez G (see Jara Facundo et al). 2003;47:457-462

\section{R}

Raab SS (see Suba and Raab). 2003;47:948-949 (Letter)

Rahmeh T (see Vesoulis et al). 2003;47:668-672

Raisanen J, Burns DK, White CL. Cytology of Subependymoma. 2003;47:518-520 (Letter)

Rajesh L, Dey P, Joshi K. Fine Needle Aspiration Cytology of Lobular Breast Carcinoma: Comparison with Other Breast Lesions. 2003;47:177-182

Rajwanshi A (see Brahmi et al). 2003;47:443-449

Rasekhi A (see Geramizadeh et al). 2003;47:701-702 (Letter)

Ravinsky E (see Jara Facundo et al). 2003;47:457-462

Reinhartz T (see Maly et al). 2003;47:1131-1134

Ribas A, Jou C, Escobar J. Ciliocythophthoria and Ameboflagellates. 2003;47:705-708 (Letter)

Rigla M (see Lerma et al). 2003;47:575-580

Rodrigues de Lima G (see Mattosinho de Castro Ferraz et al). 2003;47:154-158

Rong SD (see Pan et al). 2003;47:45-50

Roozbehi H (see Monabati et al). 2003;47:702-704 (Letter)

Rosenthal D (see Farinola et al). 2003;47:787-791

Rosenthal DL (see Ghaffar et al). 2003;47:281-286

Rosenthal E (see Oregioni et al). 2003;47:98-99 (Letter)
Rowe LR, Marshall CJ, Berry M, Larson MA, Bentz JS. Accuracy of a Slide Profiler for Endocervical Cell Detection in No-Further-Review Conventional Pap Smears. 2003;47:602-604

Rubin A. Clinical Value of Repeat Pap Smear at the Time of Colposcopy. 2003;47:698 (Letter)

Ruckait N (see Pientong et al). 2003;47:616-623

Rủžičková M (see Glosová et al). 2003;47:1087-1090

Ryška A. Multinucleated Stromal Giant Cells in Breast Lesions. 2003;47:112-113 (Letter)

\section{S}

Sáenz-Santamaría J, Catalina-Fernández I, Fernández de Mera JJ. Hydatid Cyst in Soft Tissues Mimicking Malignant Tumors: Diagnosis by Fine Needle Aspiration Cytology. 2003;47:337-340

Sáenz-Santamaría J, Catalina-Fernández I, FernándezMera JJ, Villarreal-Renedo P. Low Grade Mucoepidermoid Carcinoma Arising in Cervical Lymph Nodes: A Report of Two Cases with Fine Needle Aspiration Findings. 2003;47:470-474

Sáenz-Santamaría J, Catalina-Fernandez I, FernandezMera JJ. Sialadenitis with Crystalloid Formation: Fine Needle Aspiration Cytodiagnosis of 15 Cases. 2003;47: 1-4

Sakaki M, Hirokawa M, Sano T, Takahashi H, Tezuka K, Abe K, Sano M. Ovarian Endometriosis Showing Decidual Change and Arias-Stella Reaction with Biotin-Containing Intranuclear Inclusions. 2003;47: 321-324 (Letter)

Sakuma N (see Okamura et al). 2003;47:209-215

Salomão DR (see Kay et al). 2003;47:202-208

San Miguel P (see Ortiz-Rey et al). 2003;47:490-494

Sánchez-Fernández de la Vega J (see Hardisson et al). 2003;47:259-264

Sandweiss L (see Calvin et al). 2003;47:1139-1140 (Letter)

Sano M (see Sakaki et al). 2003;47:321-324 (Letter)

Sano T (see Sakaki et al). 2003;47:321-324 (Letter)

Sano T (see Tashiro et al). 2003;47:265-269

Santinami M (see Kalogeraki et al). 2003;47:363-367

Sanz E (see Jiménez-Heffernan et al). 2003;47:319-320 (Letter)

Saqi A (see Nassar et al). 2003;47:313-315 (Letter)

Sasagawa M, Nishino K, Honma S, Kodama S, Takahashi T. Origin of Adenocarcinoma Cells Observed on Cervical Cytology. 2003;47:410-414

Sasaki M (see Haba et al). 2003;47:88-92

Sasou S (see Kikuchi et al). 2003;47:495-500

Sato R (see Jobo et al). 2003;47:611-615

Satoh Y, Ishikawa Y, Furuta N, Tsuzuku M. Difficulty of Cytodiagnostic Approaches to Pulmonary Metastases from a Case of Low Grade Endometrial Stromal Sarcoma. 2003;47:1140-1141 (Letter) 
Sauer T (see Nygård et al). 2003;47:991-1000

Sauer T, Engh V, Holck AM, Sørpebøl G, Heim M, Furu I, Schlichting E. Imprint Cytology of Sentinel Lymph Nodes in Breast Cancer: Experience with Rapid, Intraoperative Diagnosis and Primary Screening by Cytotechnologists. 2003;47:768-773

Saurí A (see Mayayo et al). 2003;47:1116-1118

Sauvegeot J (see Studeman et al). 2003;47:605-610

Sawaya GF (see Kinney et al). 2003;47:167-171

Scheiman JM (see Afify et al). 2003;47:341-348

Schindler S (see Okonkwo et al). 2003;47:435-442

Schlichting E (see Sauer et al). 2003;47:768-773

Schneider V. CIN Prognostication: Will Molecular Techniques Do the Trick? 2003;47:115-116

Segev DL, Clark DP, Zeiger MA, Umbricht C. Beyond the Suspicious Thyroid Fine Needle Aspirate: A Review. 2003;47:709-722

Seki H (see Kobayashi et al). 2003;47:1069-1073

Selvaggi SM (see Haack and Selvaggi). 2003;47:949-950 (Letter)

Sengupta R (see Mishra et al). 2003;47:1001-1007

Sevá-Pereira T (see França et al). 2003;47:332-336

Shabaik A. Endoscopic Ultrasound-Guided Fine Needle Aspiration Cytology of Intraductal Papillary Mucinous Tumor of the Pancreas: A Case Report. 2003;47: 657-662

Sharma A (see Anand et al). 2003;47:467-469

Sharma MC (see Anand et al). 2003;47:467-469

Sharma R (see Mehdi et al). 2003;47:529-531 (Letter)

Sharma S (see Shivkumar et al). 2003;47:110-111 (Letter)

Sharma S, Mishra K, Khanna G. Fibromatosis Colli in Infants: A Cytologic Study of Eight Cases. 2003;47: 359-362

Sheikh ZA (see Pathan et al). 2003;47:299-303

Sherman Y (see Maly et al). 2003;47:1131-1134

Sherwani RK, Abrari A, Jayrajpuri ZS, Srivastava VK. Intracranial Hydatidosis: Report of a Case Diagnosed on Cerebrospinal Fluid Cytology. 2003;47:506-508

Shidham VB (see Basir et al). 2003;47:733-738

Shield PW (see Koivurinne and Shield). 2003;47:637-644

Shiratsuchi H (see Kikuchi et al). 2003;47:495-500

Shivkumar VB, Gangane N, Kishore S, Sharma S. Cytologic Features of Idiopathic Scrotal Calcinosis. 2003; 47:110-111 (Letter)

Shuangshoti S (see Tanboon et al). 2003;47:1143-1144 (Letter)

Siddiqui MT (see Allen et al). 2003;47:183-187

Siekkinen P (see Abramovich et al). 2003;47:405-409

Simões AL (see Bettini et al). 2003;47:545-549

Simões RT (see Bettini et al). 2003;47:545-549

Simsir A (see Cangiarella et al). 2003;47:965-972

Singh K (see Mishra et al). 2003;47:1001-1007

Singh N (see Arora et al). 2003;47:216-220

Singh N, Malik A, Arora VK, Bhatia A. Fine Needle Aspiration Cytology of Leprous Neuritis. 2003;47:368-372

Singh NG, Kapila K, Dawar R, Verma K. Fine Needle
Aspiration Cytology Diagnosis of Lymphoproliferative Disease of the Breast. 2003;47:739-743

Singh N, Panwar A, Masih VF, Arora VK, Bhatia A. Exploring the Links Between Quality Assurance and Laboratory Resources: An Audit-Based Study. 2003; 47:960-964

Siqueira FM (see Guimarães et al). 2003;47:1074-1076

Skare GB (see Nygård et al). 2003;47:991-1000

Skjeldestad FE (see Nygård et al). 2003;47:991-1000

Smile R (see Kumar et al). 2003;47:522-524 (Letter)

Soares EC (see França et al). 2003;47:332-336

Soares EG (see Bettini et al). 2003;47:545-549

Sobrinho LG (see Cavaco et al). 2003;47:5-12

Sodhani P (see Gupta et al). 2003;47:41-44

Sodhani P (see Gupta et al). 2003;47:795-798

Sodhani P (see Jain et al). 2003;47:381-386

Sørpebøl G (see Sauer et al). 2003;47:768-773

Soyuer I, Ekinci C, Kaya M, Genç Y, Bahar K. Diagnosis of Hepatocellular Carcinoma by Fine Needle Aspiration Cytology: Cellular Features. 2003;47:581-589

Srinivasan R (see Brahmi et al). 2003;47:443-449

Srivastava VK (see Sherwani et al). 2003;47:506-508

Stavale JN (see Mattosinho de Castro Ferraz et al). 2003; 47:154-158

Stewart JM (see Caraway et al). 2003;47:777-782

Studeman KD, Ioffe OB, Puszkiewicz J, Sauvegeot J, Henry MR. Effect of Cellularity on the Sensitivity of Detecting Squamous Lesions in Liquid-Based Cervical Cytology. 2003;47:605-610

Stulting RD (see Grossniklaus et al). 2003;47:239-246

Suba EJ, Raab SS. Population-Based Pap Screening in Cameroon. 2003;47:948-949 (Letter)

Sudilovsky D (see Baehner and Sudilovsky). 2003;47:275-280

Sugai T (see Kikuchi et al). 2003;47:495-500

Suh Y-L (see Park et al). 2003;47:624-629

Sullivan J (see Zardawi et al). 2003;47:704-705 (Letter)

Sun YT (see Pan et al). 2003;47:45-50

Sundareshan TS (see Madhumathi et al). 2003;47:101-103 (Letter)

Sundareshan TS (see Premalata et al). 2003;47:475-479

Sung H-Y (see Kinney et al). 2003;47:167-171

Sungun A (see Kabukcuoglu et al). 2003;47:311-313 (Letter)

Suzuki M (see Nagashima et al). 2003;47:1028-1032

Suzuki T (see Watanabe et al). 2003;47:99-101 (Letter)

Svobodová E (see Dušková et al). 2003;47:71-77

Swadpanich U (see Pientong et al). 2003;47:616-623

Szporn A (see Yarkin et al). 2003;47:450-456

\section{T}

Tabatabai ZL, Krishnamurthy S. Radiation-Induced Atypical Glandular Cells Mimicking Recurrent Adenocarcinoma in a Posthysterectomy Vaginal Smear. 2003;47:106-107 (Letter) 
Takahashi H (see Sakaki et al). 2003;47:321-324 (Letter)

Takahashi R (see Okuyama et al). 2003;47:696-698 (Letter)

Takahashi T (see Sasagawa et al). 2003;47:410-414

Takano T (see Kimura et al). 2003;47:1099-1102

Takatani N (see Kuwabara et al). 2003;47:515-517 (Letter)

Takei K (see Kuwabara et al). 2003;47:515-517 (Letter)

Talei AR (see Geramizadeh et al). 2003;47:701-702 (Letter)

Tan NG (see Goh et al). 2003;47:65-70

Tan PH (see Goh et al). 2003;47:65-70

Tanaka T (see Tashiro et al). 2003;47:265-269

Tanaka Y (see Hasegawa et al). 2003;47:679-684

Tanboon J, Chaipipat M, Wattanasirmkit V, Wongtabtim W, Shuangshoti S, Bunyaratavej K. Squash Cytology of Rosai-Dorfman Disease in the Sellar Region. 2003; 47:1143-1144 (Letter)

Tandon N (see Karak et al). 2003;47:227-238

Tarjan G (see Williamson et al). 2003;47:749-752

Tarrand JJ (see Caraway et al). 2003;47:777-782

Tasaki K (see Watanabe et al). 2003;47:99-101 (Letter)

Tashiro T, Hirokawa M, Iuchi K, Emoto K, Tanaka T, Monobe Y, Sano T. Cytology of Pleomorphic Lobular Carcinoma with Apocrine Cell Differentiation of the Breast: A Case Report. 2003;47:265-269

Taylor DC (see Parwani et al). 2003;47:325-331

Tezuka K (see Sakaki et al). 2003;47:321-324 (Letter)

Thamboo TP (see Wee et al). 2003;47:799-808

Thappa DM (see Kumar et al). 2003;47:522-524 (Letter)

Thomas A (see Wee et al). 2003;47:799-808

Thoresen SØ (see Nygård et al). 2003;47:991-1000

Tibbs RF, Wong JY, Logroño R. Enhancing Recovery of Endocervical Component on Gynecologic Cytology Specimens Processed by Thin-Layer Technology. 2003;47:172-176

Topal AN, Canda MS, Günel Ö. Schaumann Bodies in Bronchial and Bronchioalveolar Lavage Fluid in a Case of Sarcoidosis. 2003;47:320-321 (Letter)

Torabinezhad S (see Monabati et al). 2003;47:702-704 (Letter)

Torrinha F (see Cavaco et al). 2003;47:5-12

Tretiník P (see Dušková et al). 2003;47:71-77

Trevisan M (see França et al). 2003;47:332-336

Triggiani V, Ciampolillo A, Maiorano E. Papillary Thyroid Carcinoma, Diffuse Sclerosing Variant, with Abundant Psammoma Bodies. 2003;47:1141-1143 (Letter)

Tse SKM (see Ng et al). 2003;47:56-64

Tsuneyoshi M (see Kikuchi et al). 2003;47:495-500

Tulbah A (see Mourad et al). 2003;47:744-748

Tsuzuku M (see Satoh et al). 2003;47:1140-1141 (Letter)
Ueda M (see Kobayashi et al). 2003;47:103-106 (Letter)

Ueda M (see Kobayashi et al). 2003;47:1069-1073

Ulrich W (see Pokieser et al). 2003;47:108-110 (Letter)

Umbricht C (see Segev et al). 2003;47:709-722

Umemoto M (see Watanabe et al). 2003;47:78-82

Unni KK (see Kay et al). 2003;47:202-208

Upton MP (see Ayata et al). 2003;47:973-978

Upton MP (see Pantanowitz et al). 2003;47:1050-1054

Urabe M (see Kobayashi et al). 2003;47:309-311 (Letter)

V

Vaillo Vinagre A, Gutiérrez Martín A, Pérez Barrios A, Alberti Masgrau N, Ballestín Carcavilla C, De Agustín PDA. Fine Needle Aspiration Cytology of Neurothekeoma: A Case Report. 2003;47:485-489

Valério HMG (see França et al). 2003;47:332-336

Vardouli A (see Divani et al). 2003;47:944-945 (Letter)

Vecchione A (see Pisani et al). 2003;47:520-522 (Letter)

Veneti S, Daskalopoulou D, Zervoudis S, Papasotiriou E, Ioannidou-Mouzaka L. Liquid-Based Cytology in Breast Fine Needle Aspiration: Comparison with the Conventional Smear. 2003;47:188-192

Vera-Alvarez J, Marigil-Gómez M, García-Prats MD, Abascal-Agorreta M, López-López JI, Perez-Ruiz J. Extramedullary Plasmacytoma Presenting as a Primary Mass in the Breast: A Case Report. 2003;47:11071110

Verma K (see Kapila and Verma). 2003;47:315-317 (Letter)

Verma K (see Kapila and Verma). 2003;47:36-40

Verma K (see Kapila et al). 2003;47:555-562

Verma K (see Karak et al). 2003;47:227-238

Verma K (see Singh et al). 2003;47:739-743

Verma K, Kapila K. Salivary Gland Tumors with a Prominent Oncocytic Component: Cytologic Findings and Differential Diagnosis of Oncocytomas and Warthin's Tumor on Fine Needle Aspirates. 2003;47: 221-226

Vesoulis Z, Rahmeh T, Nelson R, Clarke R, Lu Y, Dankoff J. Fine Needle Aspiration Biopsy of Primary Renal Synovial Sarcoma: A Case Report. 2003;47:668-672

Vetrani A (see Zeppa et al). 2003;47:501-505

Viganò P (see Omodeo-Zorini et al). 2003;47:985-990

Villarreal-Renedo P (see Sáenz-Santamaría et al). 2003;47: 470-474

Volpin G (see Munichor et al). 2003;47:270-274

\section{W}

\section{U}

Uda H (see Kuwabara et al). 2003;47:515-517 (Letter)

Ueda H (see Watanabe et al). 2003;47:78-82
Waisman J (see Cangiarella et al). 2003;47:965-972

Wakasa K (see Haba et al). 2003;47:88-92

Wallace WD (see Calvin et al). 2003;47:1139-1140 (Letter) 
Wang HH (see Ayata et al). 2003;47:973-978

Wang HH (see Pantanowitz et al). 2003;47:1050-1054

Wang HL (see Lu et al). 2003;47:1008-1016

Wang N (see Wen et al). 2003;47:135-140

Wang W (see Yarkin et al). 2003;47:450-456

Warwick L (see Zardawi et al). 2003;47:704-705 (Letter)

Wasman JK (see Abramovich et al). 2003;47:405-409

Watanabe K, Ogura G, Tasaki K, Hoshi N, Suzuki T.

Fine Needle Aspiration Cytology of Inverted Sinonasal

Papilloma. 2003;47:99-101 (Letter)

Watanabe T (see Hasegawa et al). 2003;47:679-684

Watanabe T (see Ishida et al). 2003;47:1095-1098

Watanabe Y, Umemoto M, Ueda H, Nakai H, Hoshiai H, Noda K. Cytopathologic and Clinicopathologic Features of Ovarian Hepatoid Carcinoma: A Case Report. 2003;47:78-82

Wattanasirmkit V (see Tanboon et al). 2003;47:1143-1144 (Letter)

Weber KL (see Caraway et al). 2003;47:777-782

Wee A, Nilsson B. Highly Well Differentiated Hepatocellular Carcinoma and Benign Hepatocellular Lesions: Can They Be Distinguished on Fine Needle Aspiration Biopsy? 2003;47:16-26

Wee A, Thamboo TP, Thomas A. $\alpha$-FetoproteinProducing Liver Carcinomas of Primary Extrahepatic Origin: Fine Needle Aspiration Biopsy Experience in 2 Cases. 2003;47:799-808

Wen P, Abramovich CM, Wang N, Knop N, Mansbacher $S$, Abdul-Karim FW. Significance of Histiocytes on Otherwise-Normal Cervical Smears from Postmenopausal Women: A Retrospective Study of 108 Cases. 2003;47:135-140

Wenig B (see Wiriosuparto et al). 2003;47:673-678

Weynand B, Berlière M, Haumont E, Massart F, Pourvoyeur A, Bernard P, Donnez J, Galant C. A New, Liquid-Based Cytology Technique. 2003;47:149-153

White CL (see Raisanen et al). 2003;47:518-520 (Letter)

Williamson BA, DeFrias D, Gunn R, Tarjan G, Nayar R. Significance of Extensive Hyperkeratosis on Cervical/ Vaginal Smears. 2003;47:749-752

Williamsz G (see Zardawi et al). 2003;47:1077-1081

Wiriosuparto S, Krassilnik N, Gologan A, Cohen J-M, Wenig B. Malignant Fibrous Histiocytoma, Giant Cell Type, of the Breast Mimicking Metaplastic Carcinoma: A Case Report . 2003;47:673-678

Wong JY (see Tibbs et al). 2003;47:172-176

Wong WWC (see Ng et al). 2003;47:698-701 (Letter)

Wongtabtim W (see Tanboon et al). 2003;47:1143-1144 (Letter)

Wu LY (see Pan et al). 2003;47:45-50

\section{Y}

Yagata H (see Nagashima et al). 2003;47:1028-1032
Yağmur M (see Ersöz et al). 2003;47:13-15

Yalaz M (see Ersöz et al). 2003;47:13-15

Yamamoto H (see Kimura et al). 2003;47:287-292

Yamamoto M (see Kikuchi et al). 2003;47:495-500

Yan KW (see Chan et al). 2003;47:809-816

Yang S-H (see Ko et al). 2003;47:727-732

Yang YJ, Demirci SS. Evaluating the Diagnostic Significance of Nuclear Grooves in Thyroid Fine Needle Aspirates with a Semiquantitative Approach. 2003;47: 563-570

Yarkin F, Chauvin S, Konomi N, Wang W, Mo R, Bauchman G, Diaz A, Burstein D, Szporn A, Hauptman E, Zhang DY. Detection of HPV DNA in Cervical Specimens Collected in Cytologic Solution by LigationDependent PCR. 2003;47:450-456

Yip WW-L (see Ng and Yip). 2003;47:513-515 (Letter)

Yohena C (see Kinjo et al). 2003;47:663-667

Yokoyama T (see Kawahara et al). 2003;47:1127-1130

Yoshida H (see Mori et al). 2003;47:480-484

Yuan Q, Chang AR, Ng HK. Introduction of the Bethesda System to Mainland China with a Web-Based Tutorial. 2003;47:415-420

Yuenyao P (see Pientong et al). 2003;47:616-623

$\mathbf{Z}$

Zanconati F (see DellaGiustina et al). 2003;47:1017-1022

Zardawi IM, Catterall N, Duncan J, Sullivan J, Warwick L. Effects of Lubricant Gel on Conventional and LiquidBased Cervical Smears. 2003;47:704-705 (Letter)

Zardawi IM. Cellularity of Liquid-Based, Thin-Layer Cervical Cytology. 2003;47:943 (Letter)

Zardawi IM, Clark D, Williamsz G. Inflammatory Myofibroblastic Tumor of the Breast: A Case Report. 2003;47:1077-1081

Zardawi IM, Duncan J. Evaluation of a Centrifuge Method and Thin-Layer Preparation in Urine Cytology. 2003;47:1038-1042

Zeiger MA (see Segev et al). 2003;47:709-722

Zeppa P, Lepore M, Vetrani A, Palombini L. Occult Lymph Node Metastasis from Desmoplastic Small Round Cell Tumor Diagnosed by Fine Needle Aspiration Cytology: A Case Report. 2003;47:501-505

Zervoudis S (see Veneti et al). 2003;47:188-192

Zhang DY (see Yarkin et al). 2003;47:450-456

Zhang JZ, Gu M. Malignant Phyllodes Tumor of the Breast Metastatic to the Parotid Gland Diagnosed by Fine Needle Aspiration Biopsy: A Case Report. 2003; 47:253-258

Zhang WH (see Pan et al). 2003;47:45-50

Zhang X (see Pan et al). 2003;47:45-50

Zhang X, El-Sahrigy D, Elhosseiny A, Melamed MR. Simultaneous Cytomegalovirus Infection and Kaposi's Sarcoma of the Thyroid Diagnosed by Fine Needle 
Aspiration in an AIDS Patient: A Case Report and First Cytologic Description of the Two Entities Occurring Together. 2003;47:645-648
Zhu X (see Lu et al). 2003;47:1008-1016

Zoras O (see Kalogeraki et al). 2003;47:363-367

Zucoloto S (see França et al). 2003;47:332-336 